\title{
Author Index to Volume 41
}

\author{
Dentomaxillofacial Radiology (2012) 41, 709-710. doi: 10.1259/dmfr/22660345
}

Key to abbrevations: (BR) Book review, (CR) Case report, (C) Corrigendum, (E) Editorial, (Le) Letter to the editor, (R) Review, (Re) Research, (SC) Short communication, (SR) Systematic review, (TR) Technical report

\author{
Abe Y 668 (Re) \\ Ahmed M 429 (CR) \\ Akgül HM 234 (Re) \\ Aksu Y $558(\mathrm{Re})$ \\ Albarakati SF $11(\mathrm{Re})$ \\ Al-Kadi O 475 (Re) \\ Almeida SM $361(\mathrm{Re}), 553(\mathrm{Re}), 417$ \\ (Re) \\ Al-Rawi NH 197 (Re) \\ Alsadhan RI $185(\mathrm{Re})$ \\ Alsufyani NA $276(\mathrm{R})$ \\ Al-Timimi JF 197 (Re) \\ Alves-Junior SM $541(\mathrm{Re})$ \\ Alyass NS 686 (Re) \\ Anand Sherwood I 43 (Re) \\ Anbiaee N 441 (Le) \\ Anthonappa RP 444 (SR) \\ Apinhasmit W 578 (Re) \\ Arita ES $621(\mathrm{Re})$ \\ Asanuma S 411 (Re) \\ Aslan G 564 (Re) \\ Assunção Junior JNR 203 (Re) \\ Asvestas PA 328 (TR) \\ Atilgan SS $385(\mathrm{Re})$ \\ Băciuț G 548 (Re) \\ Băciuţ M 548 (Re) \\ Bóscolo FN 361 (Re), 417 (Re), 553 \\ (Re) \\ Baksi BG 285 (Re), 309 (Re) \\ Barbosa JMN 24 (Re) \\ Barra FR 396 (Re) \\ Bechara B 422 (TR) \\ Bechara BB 248 (TR) \\ Becker M 525 (CR) \\ Becker T $533(\mathrm{R})$ \\ Benson BW $356(\mathrm{Re})$ \\ Birch S 571 (Re) \\ Bogaerts R 583 (Re) \\ Bosmans H 583 (Re) \\ Bozzao A 432 (CR) \\ Brioschi ML 621 (Re) \\ Brown J 70 (TR) \\ Brunton P 267 (R) \\ Buchner A 533 (R) \\ Büyükkaplan UȘ 405 (Re), 509 (Re) \\ Çağlayan F 234 (Re), 294 (Re) \\ Çakur B 84 (CR) \\ Canger EM 259 (CR) \\ Caprioli S 432 (CR) \\ Caria PHF 103 (Re) \\ Carneiro LS 241 (Re) \\ Castillo M 340 (CR) \\ Çelenk P 259 (CR) \\ Cerçi SS 509 (Re) \\ Chang TI 691 (CR) \\ Chen R 131 ( Re) \\ Cheng G $96(\mathrm{Re})$ \\ Chikui T 18 (Re)
}

Chindasombatjaroen J 143 (Re)

Cho BH 356 (Re)

Choi S-C $117(\mathrm{Re})$

Choi Y-S 481 (Re)

Choudhary MS 696 (CR)

Christell H 571 (Re)

Christensen J 494 (Re), 662 (Re)

Correa L 203 (Re)

Costa ALF 316 (Re)

Cruz AD 316 (Re), 361 (Re), 417 (Re)

da Cunha HA 241 (Re)

da Silveira HED $136(\mathrm{Re})$

da Silveira HLD $136(\mathrm{Re})$

Dağistan S 84 (CR)

Dalili Kajan Z 3 (Re)

Davies J 30 (Re)

Dawood A 70 (TR)

de Almeida SM 316 (Re)

de Faria Vasconcelos K 64 (Re)

de Lima LP 203 (Re)

De Moraes M $103(\mathrm{Re})$

de Sousa TO 64 (Re)

de Souza Figueiredo PT 396 (Re)

Delamare EL $136(\mathrm{Re})$

Demirtas O $152(\mathrm{Re})$

Dhoore E 466 (Re)

dos Anjos Pontual A 24 (Re)

dos Anjos Pontual ML 24 (Re)

dos Anjos RF 396 (Re)

Drage NA 30 (Re)

Dua SG 436 (CR)

Durna D 84 (CR)

Dutra MEP 367 (Re)

Dutra V $136(\mathrm{Re})$

Economopoulos TL 328 (TR)

Emami M 159 (TR)

Enciso R 675 (Re)

Endo A $211(\mathrm{Re})$

Enomoto K 349 (CR)

Espagnet MCR 432 (CR)

Estrela C $64(\mathrm{Re})$

Etöz M 75 (CR)

Etöz OA 75 (CR)

Evangelista KM 64 (Re)

Everett E 489 (Re)

Fentoğlu Ö $509(\mathrm{Re})$

Fenyo-Pereira M $203(\mathrm{Re})$

Fini G 432 (CR)

Flint DJ $356(\mathrm{Re})$

Flores-Mir C 276 (R)

Fonseca da Silveira MM 24 (Re)

Frazão MAG 24 (Re)

Freire AR $103(\mathrm{Re})$

Freire JSL $24(\mathrm{Re})$

Freitas AC $396(\mathrm{Re})$

Freitas DQ $553(\mathrm{Re})$
Friedlander AH 691 (CR)

Fukami K 460 (Re)

Furukawa S 143 (Re)

Galassi S 432 (CR)

Geha H 422 (TR)

Ghoneima AA $11(\mathrm{Re})$

Gil C $367(\mathrm{Re})$

Groppo FC 103 (Re)

$\mathrm{Gu}$ Y 615 (CR)

Guan Y 131 (Re)

Guerra ENS 396 (Re)

Guldag MU 405 (Re)

Guledgud MV 305 (Re)

Gümüş BA 509 (Re)

Gupta D 169 (CR)

Haddad DS 621 (Re)

Hagström J 529 (CR)

Haiter-Neto F 553 (Re)

Haktanr A 79 (CR)

Hamada Y 460 (Re)

Harada H 349 (CR)

Harori A $294(\mathrm{Re})$

Hasegawa M 668 (Re)

Hashimoto K $18(\mathrm{Re})$

Hassan BA $645(\mathrm{Re})$

Hayakawa M 515 (TR)

Hayashi T 254 (CR)

Hedeşiu M 548 (Re), 571 (Re)

Hellén-Halme K 192 (Re), 656 (Re)

Heo M-S 117 (Re)

Hiwatashi A 601 (TR)

Hollender L 656 (Re)

Homaeieshandiz F 379 (Re)

Honda H 601 (TR)

Horner K 267 (R), 571 (Re), $583(\mathrm{Re})$

Hostens J 466 (Re)

Huang JC 481 (Re)

Huh K-H 117 (Re)

Hunter AK 217 (Re)

Ida M 594 (Re)

Idris M 429 (CR)

Imanimoghaddam M $379(\mathrm{Re})$

Imholz B 525 (CR)

Inglèse JM 649 (Re)

Inokuchi G 515 (TR)

Ishigami T 668 (Re)

Ivanauskaité D $571(\mathrm{Re})$

Iwase H 515 (TR)

Jacobs R 466 (Re), 548 (Re), 583 (Re)

Jaju PP 619 (Le)

Janda M $656(\mathrm{Re})$

Janhom A $91(\mathrm{Re})$

Jara H $630(\mathrm{Re})$

Jatti DS 520 (CR)
Johnson B $30(\mathrm{Re})$

Jørgensen PM 323 (SC)

Joshi R 211 (Re)

Jung YH 356 (Re)

Juyanda B $645(\mathrm{Re})$

Kaffe I 533 (R)

Kahn JL 649 (Re)

Kakimoto N 143 (Re)

Kamano H 601 (TR)

Kambungton J 91 (Re)

Kamburoğlu K 450 (Re)

Kami YN 18 (Re)

Kaneda T 122 (Re), 392 (Re)

Kang IW 165 (CR)

Kang J-H 481 (Re)

Kang S-H 679 (Re)

Karlo CA 637 (Re)

Katkar AS 177 (Le)

Katkar RA 177 (Le)

Kato H 703 (CR)

Kato M $122(\mathrm{Re})$

Katoh T 211 (Re)

Katz J 178 (Le)

Kavosi A 159 (TR)

Kellenberger CJ 637 (Re)

Khan M 475 (Re)

Khan N 429 (CR)

Kim J-D 224 (Re)

Kim J-H 224 (Re), 679 (Re)

Kim M-K $679(\mathrm{Re})$

Kim S-G $224(\mathrm{Re})$

Kim S-H 481 ( $\mathrm{Re})$

Kim Y-K $224(\mathrm{Re})$

King NM 444 (SR)

Kirzioğlu FY 509 (Re)

Kishino M 143 (Re)

Kobayashi I 211 (Re)

Kobayashi K 460 (Re)

Kodaira SK 367 (Re)

Kolsuz E $450(\mathrm{Re})$

Kook Y-A 481 (Re)

Kubota Y $18(\mathrm{Re})$

Kula KS 11 (Re)

Kulkarni AV 436 (CR)

Kulkarni SS 436 (CR)

Kumar LV 609 (CR)

Kurabayashi T 55 (Re), 594 (Re)

Kuribayashi A 55 (Re), 460 (Re), 594 (Re)

Ladeira DBS $417(\mathrm{Re})$

Lee GK 165 (CR)

Lee S-M $117(\mathrm{Re})$

Lee S-S 117 (Re)

Lee YH 165 (CR)

Leite AF $396(\mathrm{Re})$

Leles CR $241(\mathrm{Re})$

Lepage B 649 (Re)

Li G 373 (Re) 
Li LM 316 (Re)

Liang H 356 (Re)

Liao J 630 (Re)

Liedke GS $136(\mathrm{Re})$

Lim PF $126(\mathrm{Re})$

Lindh C $571(\mathrm{Re})$

Lith A $192(\mathrm{Re})$

Liu B $96(\mathrm{Re})$

Liu G $131(\mathrm{Re})$

Lombardi T 525 (CR)

Lopes SLPC $316(\mathrm{Re})$

Luchsinger $\mathrm{S} 466(\mathrm{Re})$

Ludlow JB $126(\mathrm{Re})$

Ma XC 373 (Re)

MacDonald DS 615 (CR)

Mah P 500 (Re)

Mahmutyazicioglu K 564 (Re)

Major PW 276 (R)

Makino Y 515 (TR)

Manika 609 (CR)

Manjula M 609 (CR)

Manjunath GS 342 (CR)

Maret D $649(\mathrm{Re})$

Markic G 637 (Re)

Matsopoulos GK 328 (TR)

Matsumura Y 703 (CR)

Matzen LH $494(\mathrm{Re})$

Mavili E 558 (Re)

McDavid WD 217 (Re), 500 (Re)

McMahan CA 248 (TR), 422 (TR)

Mehkri S 342 (CR)

Mehralizadeh S 159 (TR)

Melo NS 396 (Re)

Memon W 429 (CR)

Mendonça EF 241 (Re)

Mert A 285 (Re), 309 (Re)

Millet B 89 (Le)

Miloglu O $152(\mathrm{Re})$

Min J-H $224(\mathrm{Re})$

Mishima A $460(\mathrm{Re})$

Mittal A 169 (CR)

Miyata K $668(\mathrm{Re})$

Mohammadpour M 159 (TR)

Molnár B 328 (TR)

Monti S 432 (CR)

Moon S-C $481(\mathrm{Re})$

Moore WS 248 (TR)

Mori S $122(\mathrm{Re}), 392(\mathrm{Re})$

Moss C 89 (Le)

Müller L 637 (Re)

Murakami S $143(\mathrm{Re})$

Murat S $450(\mathrm{Re})$

Nackaerts O 548 (Re), 571 (Re)

Nagesh KS 342 (CR)

Nagumo K 55 (Re)

Nahm K-Y $481(\mathrm{Re})$

Nakamura S $55(\mathrm{Re})$

Nakanishi K 703 (CR)

Nakayama E 18 (Re)

Namiki T $668(\mathrm{Re})$

Nascimento LA 396 (Re)

Nazarinia H 159 (TR)

Neves FS $553(\mathrm{Re})$

Nguyen M 675 (Re)

Ngwenya SP 264 (CR)

Nishiyama H 254 (CR)
Nobata K $230(\mathrm{Re})$

Nobre RM 541 (Re)

Noffke CEE 264 (CR)

Noguchi H 349 (CR)

Noguchi S 349 (CR)

Nomura J 703 (CR)

Noujeim M 248 (TR), 422 (TR)

Nzima N 264 (CR)

Oda Y 254 (CR)

Ogawa H $392(\mathrm{Re})$

Ogura I $122(\mathrm{Re}), 392(\mathrm{Re})$

Okada N 55 (Re), $594(\mathrm{Re})$

Okamura K $18(\mathrm{Re})$

Okano T $211(\mathrm{Re})$

Oliveira JX 367 (Re)

Önem E 285 (Re)

Oobu K $18(\mathrm{Re})$

Özen T $450(\mathrm{Re})$

Ozonoff A 630 (Re)

Öztürk Tonguç M 509 (Re)

Pai A 440 (Le)

Pai KM 708 (Le)

Palconet G $126(\mathrm{Re})$

Pallagatti S 169 (CR)

Panchbhai AS 696 (CR)

Panmekiate S 578 (Re)

Park H-K 679 (Re)

Park W $679(\mathrm{Re})$

Patcas R 637 (Re)

Pathak N 708 (Le)

Patil K 305 (Re)

Pauwels R 583 (Re)

Payam J $645(\mathrm{Re})$

Peltomäki T $637(\mathrm{Re})$

Peters OA 649 (Re)

Petersson A 578 (Re), $656(\mathrm{Re})$

Peyre A 649 (Re)

Pinheiro JJV 541 (Re)

Pinheiro LR 541 (Re)

Polat HB 75 (CR)

Pongsiriwet $\mathrm{S} 91$ (Re)

Ponniah I 605 (CR)

Prado FB $103(\mathrm{Re})$

Prapayasatok S 91 (Re)

Prasad RS 440 (Le)

Prietsch JR 136 (Re)

Pulcini F 432 (CR)

Purkayastha S 70 (TR)

Qu XM $373(\mathrm{Re})$

Rabie ABM 444 (SR)

Rahrooh M 379 (Re)

Raina A 305 (Re)

Raitz R 203 (Re)

Raja JV 475 (Re)

Rajiah D 605 (CR)

Rajkumar GC 342 (CR)

Rakgwale NB 264 (CR)

Ramachandra VK 475 (Re)

Rathore S 489 (Re)

Raubenheimer EJ 264 (CR)

Reddy SJ 520 (CR)

Reeves TE 500 (Re)

Ribeiro ALR 541 (Re)
Ribeiro DA 181 (R)

Rodrigues CD $64(\mathrm{Re})$

Rodrigues Pinheiro M das G 541 (Re)

Rohlin M 571 (Re)

Romano A 432 (CR)

Rossi AC 103 (Re)

Sahman H 75 (CR)

Söğüt Ö 285 (Re)

Sümbüllü MA 84 (CR), 234 (Re)

Sahman H 558 (Re)

Saito M 254 (CR)

Saito N $630(\mathrm{Re})$

Saitoh H 515 (TR)

Sakai O $630(\mathrm{Re})$

Sakata H 411 (Re)

Sakayanagi M $122(\mathrm{Re})$

Sakuma A 515 (TR)

Sanderink GCH 373 (Re)

Santos KCP 367 (Re)

Sasaki T 55 (Re)

Sato K $411(\mathrm{Re})$

Sauret-Jackson V 70 (TR)

Savranlar A $564(\mathrm{Re})$

Schou S 494 (Re)

Schulze R 1 (E), 355 (E), 443 (E)

Schropp L 298 (Re), 686 (Re)

Scolozzi P 525 (CR)

Sedghizadeh PP $675(\mathrm{Re})$

Şekerci AE 75 (CR), 152 (Re), 558 (Re)

Sekiya K 392 (Re)

Sen BH $285(\mathrm{Re})$

Serman N 620 (Le)

Sethurajan SB 605 (CR)

Shahab S 159 (TR)

Shailaja SR 609 (CR)

Sheikh S 169 (CR)

Shelley AM 267 (R)

Shet T $436(\mathrm{CR})$

Shetty NS 436 (CR)

Shi H 37 (Re)

Shibuya H 349 (CR)

Shima T $230(\mathrm{Re})$

Shimamoto H 143 (Re)

Shingaki S 254 (CR)

Shiozaki K $460(\mathrm{Re})$

Shirakawa T 411 (Re)

Sidana S 89 (Le)

Silva MAG $64(\mathrm{Re})$

Sisman Y $152(\mathrm{Re}), 558(\mathrm{Re})$

Sixou M 649 (Re)

Soğur E 309 (Re)

Souza TC 553 (Re)

Spin-Neto R 298 (Re)

Stavropoulos A 298 (Re), $686(\mathrm{Re})$

Stavropoulos F 178 (Le)

Stecke J 361 (Re)

Steinberg M $630(\mathrm{Re})$

Strehle E-M 175 (CR)

Suh KJ 165 (CR)

Sujir N 708 (Le)

Suomalainen A 529 (CR)

Sur J $211(\mathrm{Re})$

Törnwall J 529 (CR)

Tabuchi T 668 (Re)

Tafakhori Z 379 (Re), 441 (Le)
Tagawa T 703 (CR)

Tanner JM 691 (CR)

Taromsari M 3 (Re)

Taufiq M 429 (CR)

Telmon N 649 (Re)

Tetsumura A 55 (Re), 594 (Re)

Theodorakou C 583 (Re)

Tian Z $110(\mathrm{Re})$

Tokmak TT $558(\mathrm{Re}), 152(\mathrm{Re})$

Tolinov V 175 (CR)

Tozoğlu U 294 (Re)

Treil J 649 (Re)

Tsukioka T 392 (Re)

Tuji FM 541 (Re)

Tyndall D 489 (Re)

Tyndall DA $126(\mathrm{Re})$

Uchino S 349 (CR)

Ullrich O $637(\mathrm{Re})$

Uthman AT 197 (Re)

Uzun L 564 (Re)

Vaeth M 494 (Re), 662 (Re)

van der Stelt P 645 (Re)

Vandenberghe B 466 (Re)

Vargas MC 340 (CR)

Vizzotto MB 136 (Re)

Walker A $583(\mathrm{Re})$

Wang P 110 (Re)

Wang S $37(\mathrm{Re})$

Wang S-P $96(\mathrm{Re})$

Wang Y-N $96(\mathrm{Re})$

Watanabe H 55 (Re)

Wenzel A 298 (Re), 323 (SC), 494

(Re), $662(\mathrm{Re}), 686(\mathrm{Re})$

Wesselink PR $645(\mathrm{Re})$

Windisch P 328 (TR)

Wright JT 489 (Re)

Wu S $131(\mathrm{Re})$

Yüksel S $450(\mathrm{Re})$

Yabuuchi H 18 (Re)

Yajima D 515 (TR)

Yaman F 385 (Re)

Yamashita H 349 (CR)

Yamashita K 601 (TR)

Yanase S 703 (CR)

Yang J 110 (Re)

Yazgan H $564(\mathrm{Re})$

Yi W-J 117 (Re)

Yilmaz AB 234 (Re), 152 (Re)

Yilmaz UN 385 (Re)

Yoshida M 349 (CR)

Yoshino N $55(\mathrm{Re})$

Yoshiura K 18 (Re)

Yoshiura T 601 (TR)

Yu Q 37 (Re), $110(\mathrm{Re})$

Yun P-Y 224 (Re)

Yura S $230(\mathrm{Re})$

Zahedanaraki S 379 (Re)

Zain-Alabdeen EH 185 (Re)

Zhang L 615 (CR)

Zhang ZY 373 (Re)

Zhao Y 96 (Re) 